ARTICLE

\title{
Probing the heterogeneous structure of eumelanin using ultrafast vibrational fingerprinting
}

\author{
Christopher Grieco ${ }^{1}$, Forrest R. Kohl ${ }^{1}$, Alex T. Hanes ${ }^{1} \&$ Bern Kohler (D) ${ }^{1 凶}$
}

Eumelanin is a brown-black biological pigment with sunscreen and radical scavenging functions important to numerous organisms. Eumelanin is also a promising redox-active material for energy conversion and storage, but the chemical structures present in this heterogeneous pigment remain unknown, limiting understanding of the properties of its lightresponsive subunits. Here, we introduce an ultrafast vibrational fingerprinting approach for probing the structure and interactions of chromophores in heterogeneous materials like eumelanin. Specifically, transient vibrational spectra in the double-bond stretching region are recorded for subsets of electronic chromophores photoselected by an ultrafast excitation pulse tuned through the UV-visible spectrum. All subsets show a common vibrational fingerprint, indicating that the diverse electronic absorbers in eumelanin, regardless of transition energy, contain the same distribution of IR-active functional groups. Aggregation of chromophores diverse in oxidation state is the key structural property underlying the universal, ultrafast deactivation behavior of eumelanin in response to photoexcitation with any wavelength.

\footnotetext{
${ }^{1}$ Department of Chemistry and Biochemistry, The Ohio State University, 100 West 18th Avenue, Columbus, Ohio 43210, USA

凶email: kohler@chemistry.ohio-state.edu
} 
$\mathrm{M}$ elanins are multifunctional, biological pigments found in virtually every organism. Melanin pigments protect cells from damage by sunlight and by oxidants, but melanins are also associated with major human diseases. The brown-black pigment known as eumelanin plays an important, but uncertain role in melanoma ${ }^{1,2}$, whereas the chemically similar neuromelanin is present at greatly reduced levels in the brains of patients with Parkinson's disease ${ }^{3}$. Very recently, eumelaninbased materials have attracted widespread interest for solar energy conversion, electrochemical energy storage, and biomedical applications ${ }^{4-6}$. Because it absorbs broadly from the UV to near-infrared spectrum and because of its ability to conduct ${ }^{7,8}$ and store charge, eumelanin is receiving considerable attention for applications including photocatalysis, battery electrodes, and supercapacitors 5 .

Despite several decades of study, the chemical structure of eumelanin remains elusive and the nature and assembly motifs of its light-absorbing units, or chromophores, are unknown. Understanding of the fundamental chromophores and their interactions is needed to explain eumelanin's broad and featureless absorption spectrum (Fig. 1a) and its excited state dynamics. This knowledge gap not only impedes understanding of the role eumelanin plays in human disease, but also hinders the rational design of functional materials for energy and biomedical applications ${ }^{9}$.

The chemical disorder model proposed by Tran et al. ${ }^{10}$ postulates that the broad and featureless absorption spectrum of eumelanin is a superposition of absorption bands owing to chemically heterogeneous subunits. Transient electronic spectral hole burning observed in femtosecond laser experiments on eumelanins $^{11,12}$ (Fig. 1b) indicates the presence of distinct chromophores that differ in their absorption spectra. Although these results confirm that eumelanin is a heterogeneous ensemble of chromophores with different lowest-energy electronic transitions, the microscopic properties that differentiate the chromophores and determine their excited state behaviors remain highly uncertain.

Eumelanin is thought to be built from the indolic compounds, 5,6-dihydroxyindole (DHI) and 5,6-dihydroxyindole-2-carboxylic acid (DHICA) (Fig. 1c). Covalently linking these building blocks into protomolecules ${ }^{13}$ tunes their conjugation lengths but preserves their heteroatom-based functional groups (Fig. 1d-e). Importantly, electronic conjugation can extend across multiple indolic groups, depending on redox state (Fig. 1d). The protomolecules likely include a variety of redox-mutable subunits such as hydroquinone, quinone methide, quinone imine, and quinone (Fig. 1e). Noncovalent interactions are thought to further modulate the structure of eumelanin, and a currently favored paradigm is that planar eumelanin protomolecules assemble into nanoaggregates through $\pi-\pi$ interactions ${ }^{13-15}$. These aggregates are likely to be heterogeneous not only in terms of their packing structure, but also because they may contain protomolecules that differ in chemical structure and/or redox state.

Investigators have augmented the chemical disorder model by pointing out that electronic conjugation length ${ }^{16}$, redox state variations ${ }^{16-20}$, and excitonic delocalization ${ }^{14,15}$ could all affect the spectral and dynamical properties of eumelanin chromophores, but to an unknown degree. Although it is currently difficult to disentangle these effects, the observation of spectral hole burning ${ }^{11}$ in electronic transient absorption experiments (e.g., Fig. 1b) suggests that different subsets of chromophores in eumelanin can be interrogated spectroscopically. Coupling transient spectral hole burning to a structurally sensitive measurement, such as vibrational spectroscopy, would allow electronic transition energies to be correlated with IR-active functional groups like carbonyls, which are expected to be present in higher amounts in more oxidized protomolecules.

Here, we combine the recently demonstrated ability to selectively excite eumelanin chromophores based on their transition energies $^{11}$ with time-resolved vibrational spectroscopy, a technique that has not been used previously to study any form of melanin. By tuning an excitation pulse from the UV through the visible, subsets of electronic chromophores are selected, and a mid-IR probe pulse detects transient changes in vibrational modes in an approach we term ultrafast vibrational fingerprinting (UVF). This technique, which provides much of the information content of a two-dimensional electronic-vibrational spectroscopy (2D EV) experiment, is used to investigate whether

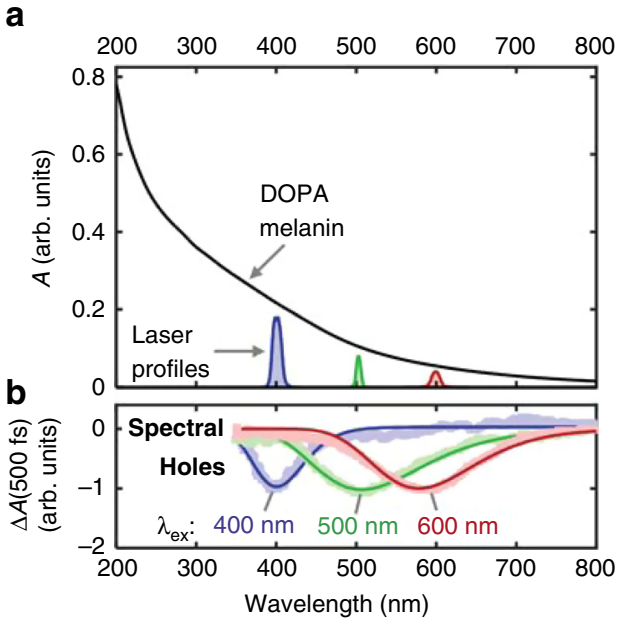

C Eumelanin building blocks
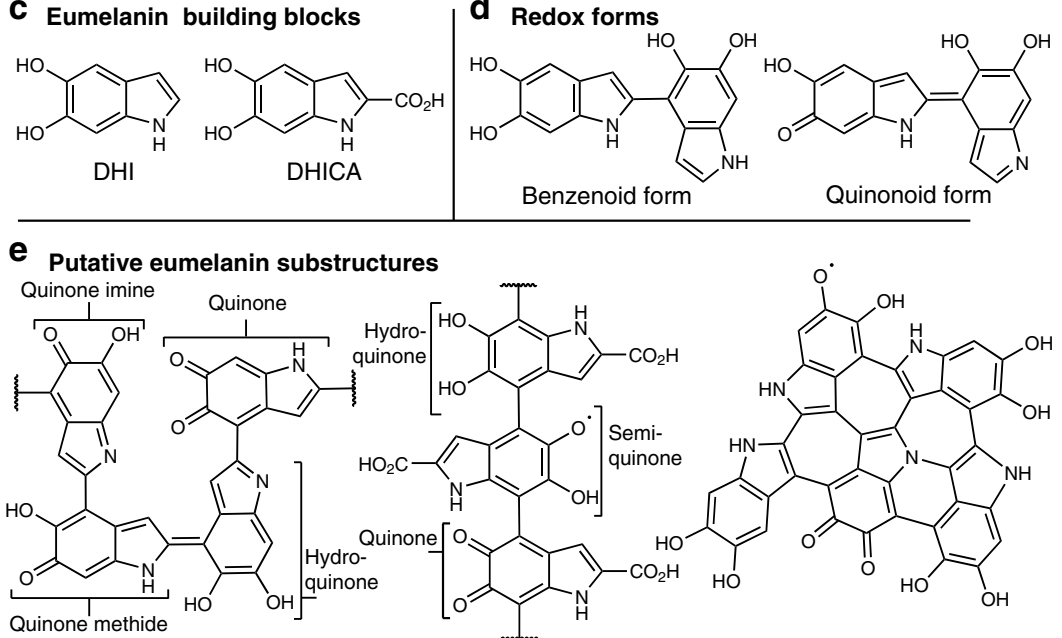

DHI-derived domain

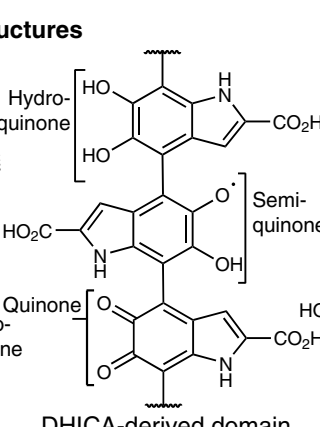

DHICA-derived domain

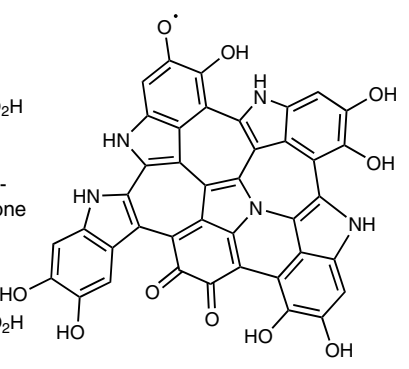

2-dimensional sheet

Fig. 1 Transient spectral hole burning of DOPA melanin (eumelanin) and putative chromophore structures. a Absorption spectrum (black curve) of an aqueous solution of DOPA melanin. Profiles of excitation laser pulses (colored curves) used in femtosecond transient absorption measurements showing their spectral width (arbitrary vertical scaling). b Transient spectral holes extracted from transient absorption spectra measured 500 fs after excitation at 400,500 , or $600 \mathrm{~nm}$. c Chemical structures of eumelanin building blocks. d Dimers of the building blocks, showing two major types of redox forms. e A few substructures proposed for DOPA melanin and other eumelanins. Labels indicate redox forms, which vary from least to most oxidized in the order hydroquinone<semiquinone<quinone imine/methide<quinone. 
chromophores that absorb at different wavelengths differ in the number and kinds of IR-active functional groups by probing their double-bond stretching modes. Each subset of selected chromophores has the same transient vibrational spectrum, or fingerprint, in the measurement window spanning $1300-1900 \mathrm{~cm}^{-1}$ and identical decay kinetics. This suggests that each subset of electronic chromophores contains the same distribution of functional groups as any other set, a property that we propose underlies the universal photoresponse observed in eumelanin ${ }^{11}$.

\section{Results}

Demonstrating UVF. UVF (see "Discussion" of this method below) was used to correlate electronic and vibrational degrees of freedom in cyclohexane (solvent) mixtures of the catechol (Cat) and quinone (Quin) derivatives with the structures shown in Fig. 2a. These molecules model the dihydroxy and quinone functional groups present in eumelanin ${ }^{21}$. Cat and Quin selfassociate to yield hydrogen-bonded heterodimers (Fig. 2b) when their concentrations in cyclohexane are sufficiently high ${ }^{21}$. Here, we study both a dilute (Fig. 2a, c, e, g) and a concentrated (Fig. 2b, $\mathrm{d}, \mathrm{f}, \mathrm{h})$ mixture.

UV-visible (UV-Vis) absorption spectra (Fig. 2c, d) and FTIR spectra (Fig. 2g, h) were recorded of Cat+Quin mixtures and Catonly and Quin-only control samples. The UV-vis spectrum of the dilute mixture (Fig. 2c) approximately equals the sum of the

a<smiles>CC(C)(C)C1=CC(=O)C(=O)C(C(C)(C)C)=C1</smiles>

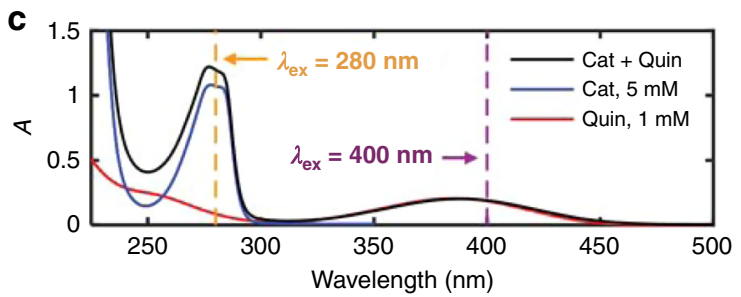

e

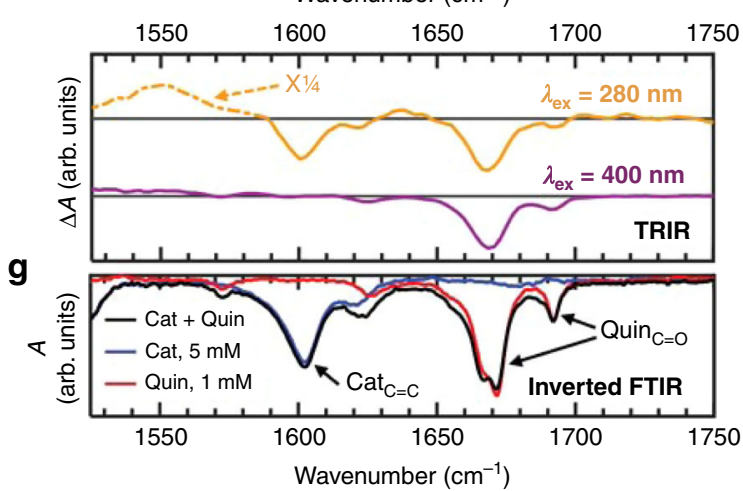

spectra of the Cat-only and Quin-only solutions, which have lowest energy bands at $280 \mathrm{~nm}$ and $387 \mathrm{~nm}$, respectively. For the spectrum of the concentrated mixture (Fig. 2d), the $387 \mathrm{~nm}$ band due to Quin broadens and redshifts to $400 \mathrm{~nm}$, which results from hydrogen bonding between Cat and Quin molecules ${ }^{21}$.

The FTIR spectra of the Cat-only and Quin-only solutions are essentially independent of concentration (Fig. 2g, h). The Catonly solutions feature a prominent vibrational band at $1603 \mathrm{~cm}^{-1}$ (aromatic $\mathrm{C}=\mathrm{C}$ stretching), whereas the Quin-only solutions feature major bands at $1672 \mathrm{~cm}^{-1}$ and $1692 \mathrm{~cm}^{-1}(\mathrm{C}=\mathrm{O}$ stretching). In the dilute mixture, there are minimal changes in the peak positions and lineshapes of the $\mathrm{C}=\mathrm{C}$ and $\mathrm{C}=\mathrm{O}$ bands (Fig. 2g). In the concentrated mixture, the $\mathrm{C}=\mathrm{O}$ bands of Quin downshift to $1665 \mathrm{~cm}^{-1}$ and $1688 \mathrm{~cm}^{-1}$ and change significantly in lineshape (Fig. 2h) owing to hydrogen bonding between Cat and Quin molecules. Each reported vibrational frequency corresponds to the frequency of maximum intensity for the relevant band.

Figure $2 \mathrm{e}, \mathrm{f}$ show the time-resolved infrared (TRIR) spectra of the Cat+Quin mixtures averaged over 1-2 ps. The dilute mixture was excited either at $280 \mathrm{~nm}$, where Cat and Quin molecules absorb, or at $400 \mathrm{~nm}$, where only Quin molecules absorb (dashed vertical lines in Fig. 2c). Exciting at $280 \mathrm{~nm}$ produces negative peaks in the transient absorption $(\Delta A)$ spectrum at $1600 \mathrm{~cm}^{-1}$, $1668 \mathrm{~cm}^{-1}$, and $1693 \mathrm{~cm}^{-1}$ (orange trace in Fig. 2e), which are close in frequency to ones measured in the FTIR spectra of Cat,

b
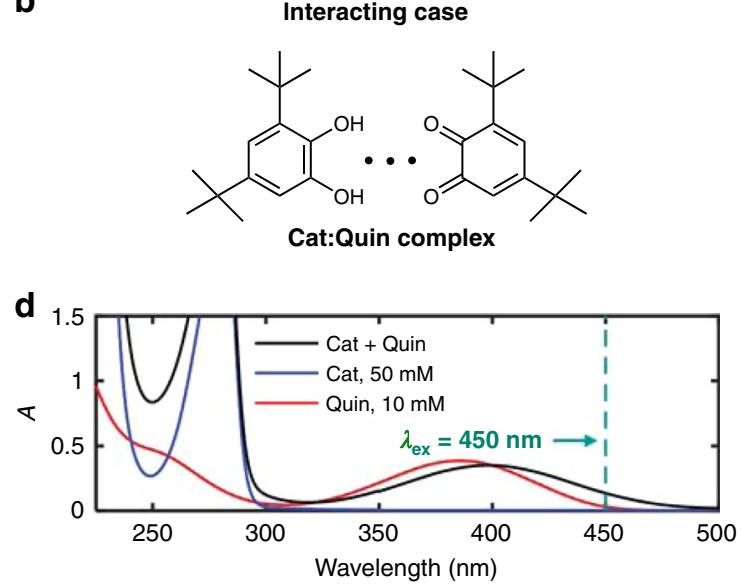

f

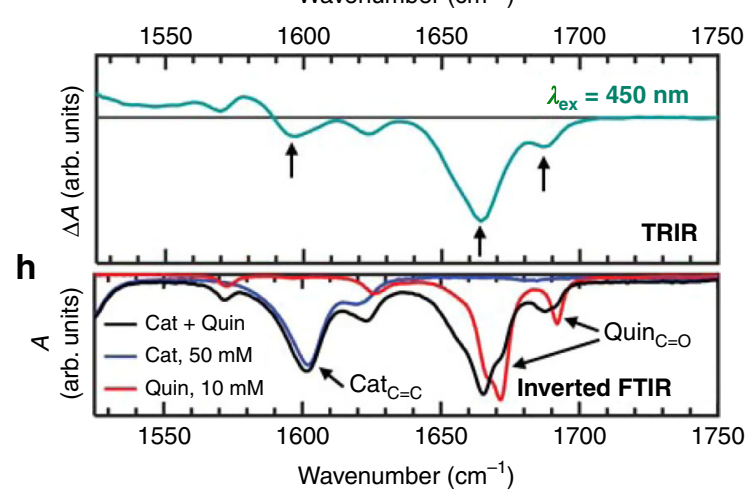

Fig. 2 Ultrafast vibrational fingerprinting demonstrated for a model system. Solutions of catechol (Cat) and quinone (Quin) mixtures in cyclohexane contain either a non-interacting, isolated molecules, or $\mathbf{b}$ molecules interacting through intermolecular hydrogen bonds. The Cat:Quin mole ratios were fixed at 5:1. a, b Chemical structures of Cat and Quin. c, d UV-vis spectra of cyclohexane solutions of Cat, Quin, and their mixtures, highlighting the excitation wavelengths used in time-resolved infrared (TRIR) spectroscopy experiments. The cell pathlengths used in $\mathbf{c}$ and $\mathbf{d}$ were $1 \mathrm{~mm}$ and $200 \mu \mathrm{m}$, respectively. e, $\mathbf{f}$ TRIR spectra of the mixture solutions averaged from 1-2 ps, recorded at the indicated excitation wavelengths. $\mathbf{g}$, $\mathbf{h}$ Inverted FTIR spectra of cyclohexane solutions of Cat, Quin, and their mixtures. 
a

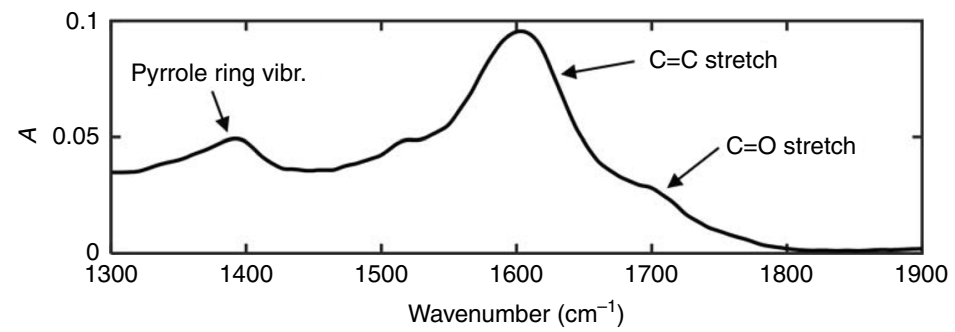

b

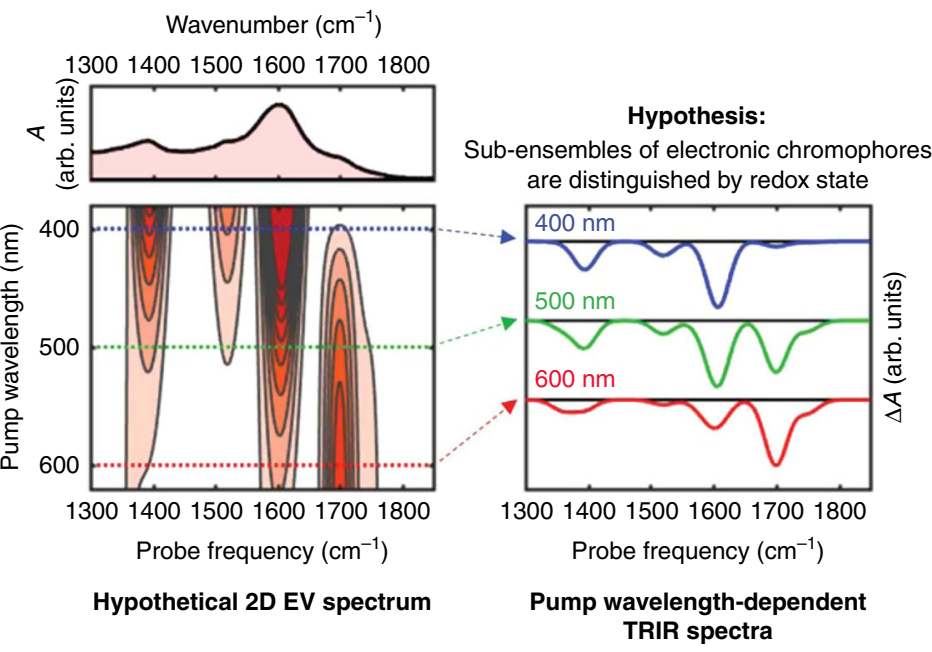

Fig. 3 Vibrational spectroscopy of DOPA melanin and UVF predictions. a FTIR spectrum of DOPA melanin dissolved in phosphate-buffered $\mathrm{D}_{2} \mathrm{O}$, including vibrational assignments. b Ultrafast vibrational fingerprinting (UVF) spectroscopy vs. two-dimensional electronic-vibrational (2D EV) spectroscopy for a hypothetical sample containing multiple electronic chromophores that can be distinguished by the redox state of their oxidizable IRactive functional groups. With time-resolved infrared (TRIR) spectroscopy (right), a subensemble of eumelanin chromophores is photoselected using a particular visible wavelength and its time-resolved vibrational spectrum is measured. These spectra are essentially horizontal slices through the hypothetical 2D EV spectrum (left). The 2D signals are assumed to only contain negative absorptive (bleaching) contributions for simplicity. Note that the presence of positive signal contributions from new vibrational frequencies in excited electronic states can alter the positions of the negative bands.

Quin, and their dilute mixture (Fig. 2g). Exciting at $400 \mathrm{~nm}$ produces negative peaks at $1668 \mathrm{~cm}^{-1}$ and $1693 \mathrm{~cm}^{-1}$, which are close in frequency to peaks in the Quin FTIR spectrum (red trace in Fig. $2 \mathrm{~g}$ ), but not at $1600 \mathrm{~cm}^{-1}$, the frequency of the band assigned to Cat. The TRIR spectrum of the concentrated mixture excited at $450 \mathrm{~nm}$ (dashed vertical line in Fig. 2d) has negative peaks at $1664 \mathrm{~cm}^{-1}$ and $1688 \mathrm{~cm}^{-1}$, and, most significantly, at $1597 \mathrm{~cm}^{-1}$ (see arrows in Fig. 2f). These peaks closely match ones of Cat and Quin in the FTIR spectrum of the concentrated Cat+Quin mixture (black trace in Fig. 2h).

Electronic-vibrational correlations in DOPA melanin. The FTIR spectrum of the synthetic eumelanin, DOPA melanin (Fig. 3a), shows several bands or shoulders in the $1300-1900 \mathrm{~cm}^{-1}$ region. The most prominent features at $1391 \mathrm{~cm}^{-1}, 1604 \mathrm{~cm}^{-1}$, and $1706 \mathrm{~cm}^{-1}$ are labeled in Fig. 3a by mode assignments for natural Sepia melanin reviewed by Centeno and Shamir ${ }^{22}$. Fig. 3b depicts hypothetical correlations between the visible absorption spectra and the vibrational spectra of DOPA melanin, based on the hypothesis that electronic chromophores are differentiated by IR-active functional groups. Importantly, the vibrational frequencies of double-bond stretching modes such as the $\mathrm{C}=\mathrm{O}$, $\mathrm{C}=\mathrm{N}, \mathrm{C}=\mathrm{C}$, and ring stretching modes probed here are very sensitive to the oxidation state of the parent compound as illustrated by calculations for the various redox forms of $\mathrm{DHI}^{23}$. If chromophores that absorb at longer wavelengths in the electronic absorption spectrum are more oxidized, then bleaching of the relatively high-frequency $\mathrm{C}=\mathrm{O}$ stretching modes of their quinone groups should be prominent (red trace in Fig. 3b), whereas if blue- absorbing electronic chromophores are more reduced, then minimal or no $\mathrm{C}=\mathrm{O}$ stretching might be detected (blue trace in Fig. 3b).

Normalized TRIR spectra of a DOPA melanin solution recorded 1 ps after photoexcitation at $265 \mathrm{~nm}, 300 \mathrm{~nm}, 400 \mathrm{~nm}$, $500 \mathrm{~nm}$, or $600 \mathrm{~nm}$ are identical within experimental uncertainty (Fig. 4, also see Supplementary Note 1). Each TRIR spectrum in Fig. 4 nearly mirrors the FTIR spectrum and exhibits negativegoing peaks located at similar frequencies (see the dashed vertical lines in Fig. 4). The TRIR peak frequencies do not vary as the excitation wavelength is changed.

Excited state relaxation kinetics in DOPA melanin. Exciting DOPA melanin at $265 \mathrm{~nm}$ produces the TRIR spectra in Fig. 5a. All other tested excitation wavelengths produce similar spectral behavior (Supplementary Note 2). The negative-going bands are already present at $0.5 \mathrm{ps}$ and then decay within $15 \mathrm{ps}$. A concurrent decay is seen for overlapping broad, positive $\Delta A$ signals spanning the entire spectral window. After $15 \mathrm{ps,} \mathrm{the} \mathrm{spectra} \mathrm{no}$ longer resemble the inverted FTIR spectrum of DOPA melanin, but instead agree with the differential absorption spectrum of heated $\mathrm{D}_{2} \mathrm{O}$ relative to its room temperature spectrum (Fig. $5 \mathrm{c}$ and Supplementary Note 3 ). The evolution of the vibrational feature in the TRIR spectrum near $1600 \mathrm{~cm}^{-1}$ is more clearly seen in the two-dimensional map in Fig. 5b. The white line in this map shows the evolution of the negative peak, which shifts from $1627 \mathrm{~cm}^{-1}$ (DOPA melanin signal) to $1602 \mathrm{~cm}^{-1}$ (hot $\mathrm{D}_{2} \mathrm{O}$ signal).

As explained in detail in Supplementary Note 4, the TRIR spectra can be modeled by contributions from DOPA melanin 
excited states, $\mathrm{D}_{2} \mathrm{O}$ molecules that are heated by accepting vibrational energy following excited state decay by DOPA melanin, and a spectrally broad background signal (see Supplementary Note 5). Importantly, the kinetics of both solvent

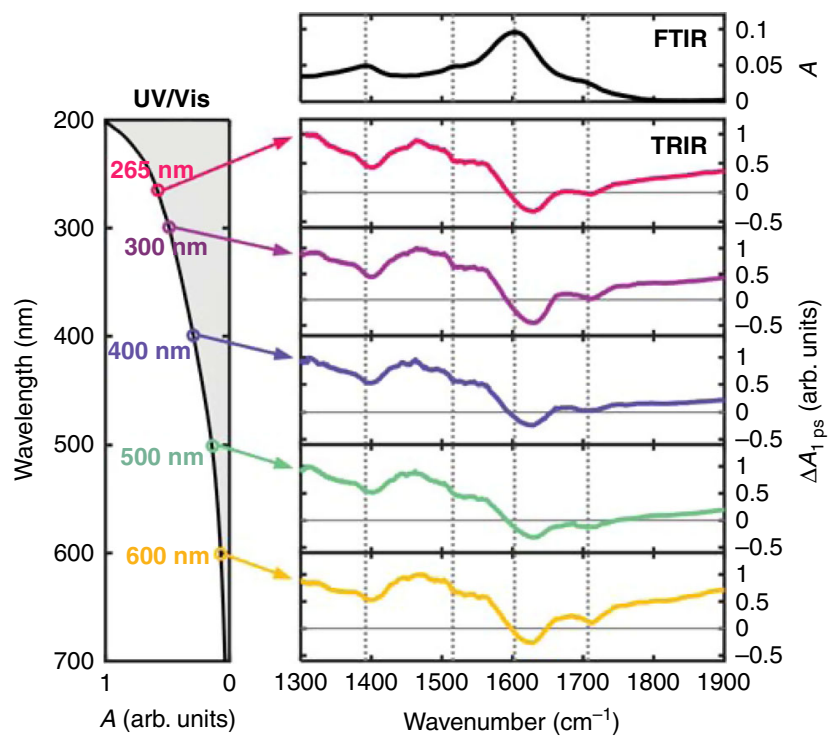

Fig. 4 Ultrafast vibrational fingerprinting of DOPA melanin. Time-

resolved vibrational spectra (TRIR) are shown at $1 \mathrm{ps}$ after photoexcitation at the wavelengths indicated on the left. Spectra are normalized for clarity. Vertical dotted lines indicate ground state vibrational peak positions. The electronic (UV-vis) and vibrational (FTIR) absorption spectra of DOPA melanin are shown on the left and top, respectively, with axes that are linear in absorbance. heating and excited state decay can be extracted from the data without the need for a kinetic model by least-squares fitting to the basis spectra shown by the red and blue curves in Fig. $5 \mathrm{c}$. The resulting kinetics obtained from global fitting are shown in Fig. 5 d. The hot $\mathrm{D}_{2} \mathrm{O}$ signal grows exponentially with a time constant of $2.8 \pm 0.4$ ps (95\% confidence interval estimated from the fit shown by the solid black curve in Fig. $5 \mathrm{~d}$ ).

The normalized TRIR kinetics of DOPA melanin probed at $1310 \mathrm{~cm}^{-1}$ are the same for all excitation wavelengths used in this study (Fig. 5e). The kinetics are also independent of excitation fluence when normalized at $1 \mathrm{ps}$ (Fig. 5f). Note that the TRIR decay kinetics probed at $1310 \mathrm{~cm}^{-1}$ are devoid of signals from hot $\mathrm{D}_{2} \mathrm{O}$ because there is an isosbestic point in the temperaturedependent $\mathrm{D}_{2} \mathrm{O}$ FTIR spectrum at this frequency (see Supplementary Note 3).

\section{Discussion}

TRIR spectroscopy with UV-vis excitation and mid-infrared probing is used in this study to fingerprint the chromophores in DOPA melanin by monitoring IR-active vibrations that change in response to photoexcitation. For aromatic molecules, photoexcitation typically produces $\pi \pi^{*}$ and $n \pi^{*}$ excited states that are delocalized over the full nuclear framework. This causes bleaching of virtually all double-bond vibrational bands in the electronic ground state owing to frequency shifts upon excitation ${ }^{24}$. In a heterogeneous sample like eumelanin, subsets of absorbers can be selectively photoexcited by tuning the excitation wavelength ${ }^{11}$. In the language of hole burning spectroscopy, photoexcitation excites an isochromat of molecules that have a common ability to absorb the pump wavelength ${ }^{25}$. Detecting the IR frequencies bleached by the excitation pulse can provide structural insights through information about the types of functional groups present in the isochromat. We refer to the use of pump a

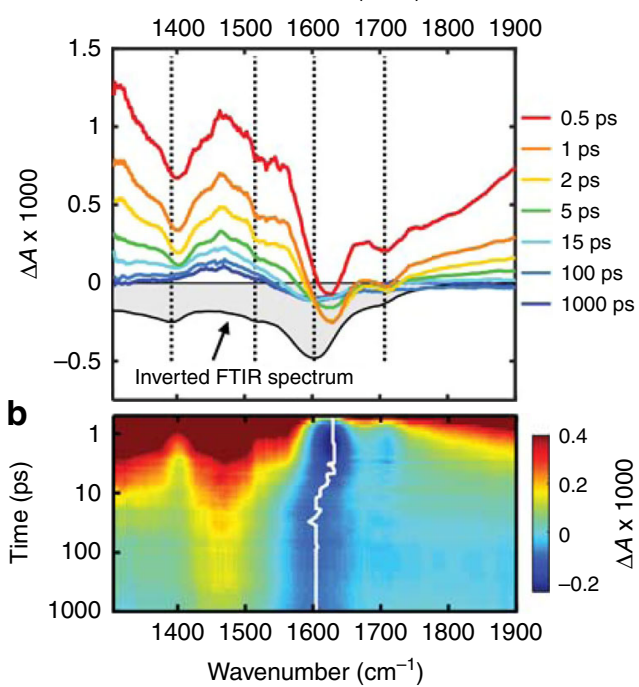

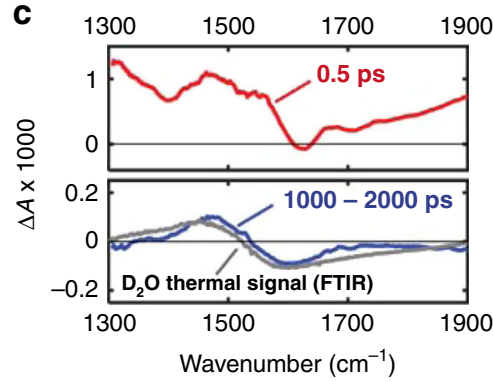

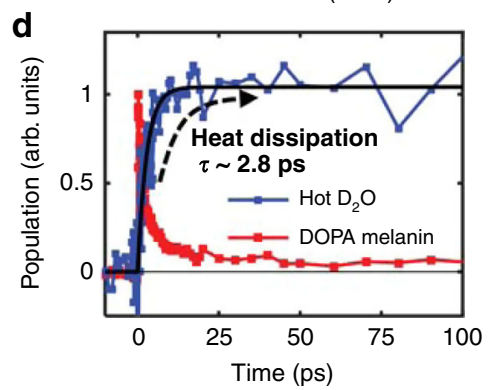

e

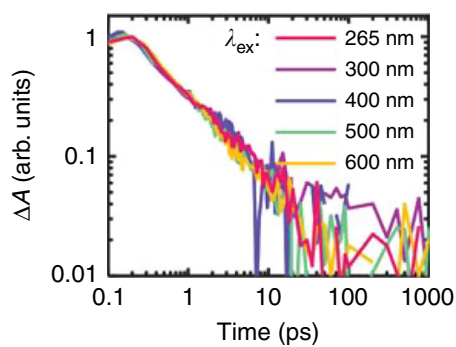

f

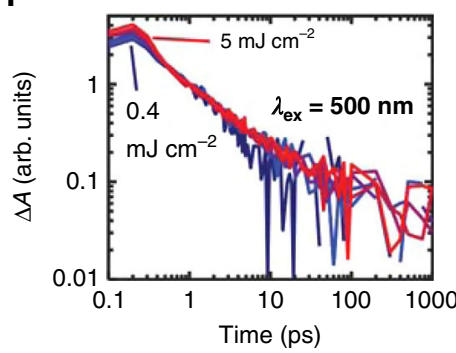

Fig. 5 Time-resolved infrared (TRIR) spectra and kinetics of DOPA melanin. a Transient absorption spectra of a solution of DOPA melanin dissolved in phosphate-buffered $\mathrm{D}_{2} \mathrm{O}$, shown at several time delays after $265 \mathrm{~nm}$ excitation. Vertical dotted lines indicate ground state absorption peak positions. $\mathbf{b}$ The corresponding two-dimensional transient absorption map, showing $\Delta A \times 1000$ with a colormap from -0.25 (blue) to 0.4 (red). The white trace shows the kinetics of the minimum signal intensity of the transient absorption spectrum. c Basis spectra selected for spectral modeling. The gray spectrum shows the $\mathrm{D}_{2} \mathrm{O}$ thermal signal estimated using temperature-dependent FTIR spectra (see Supplementary Fig. 4). d Isolated kinetics of the DOPA melanin signal compared with the $\mathrm{D}_{2} \mathrm{O}$ thermal signal. The black trace is a fit to the latter with an exponential growth model, and the corresponding time constant $(\tau)$ is shown. e Normalized excitation wavelength-dependent transient absorption kinetics of DOPA melanin probed at $1310 \mathrm{~cm}^{-1}$. $\mathbf{f}$ Power-dependent kinetics probed at $1310 \mathrm{~cm}^{-1}$ and recorded using $500 \mathrm{~nm}$ excitation, normalized at $1 \mathrm{ps}$. Seven traces are shown with pump pulse fluences that vary between 0.4 and $5.0 \mathrm{~mJ} \mathrm{~cm}^{-2}$. 
wavelength-dependent TRIR spectroscopy for identifying the vibrational signatures of diverse chromophores selected by their transition energies as UVF.

UVF provides some of the same information correlating electronic and vibrational degrees of freedom as two-dimensional electronic-vibrational (2D EV) spectroscopy ${ }^{26-28}$, but is easier to implement. In the frequency-domain realization of $2 \mathrm{D}$ spectroscopy, also known as the hole burning method ${ }^{29,30}$, a pump pulse with a bandwidth less than the homogeneous linewidth of an electronic transition of interest excites the sample and burns a hole in its spectrum, and a transient or pump-probe spectrum is recorded. Repeating this procedure at many excitation wavelengths produces a two-dimensional spectrum. Our pump wavelength-dependent TRIR measurements constitute a frequency-domain implementation of $2 \mathrm{D} \mathrm{EV}$ spectroscopy in which the TRIR spectra are slices ${ }^{31}$ through the full $2 \mathrm{D}$ EV spectrum (see Fig. 3b).

Tuning the excitation wavelength selects distinct subsets of absorbers $^{11}$, yet the vibrational fingerprints recorded for isochromats excited from $265 \mathrm{~nm}$ to $600 \mathrm{~nm}$ are indistinguishable within experimental uncertainty (Fig. 4). This observation disproves the hypothesis that eumelanin chromophores absorbing at different wavelengths can be differentiated based on their oxidizable, IR-active functional groups. Possible explanations for how a constant vibrational response can result, even as the electronic transition energy is tuned, differ depending on the degree of interaction among the chromophores as we discuss next.

Non-interacting electronic absorbers that differ in their vibrational spectra would yield distinct fingerprints as seen in the dilute Cat + Quin mixture. In this case, a $400 \mathrm{~nm}$ excitation pulse excites only the oxidized chromophore, Quin, bleaching its $\mathrm{C}=\mathrm{O}$ bands, but not the $\mathrm{C}=\mathrm{C}$ bands of Cat (Fig. 2e). Thus, in the absence of interactions, the observation of identical fingerprints in DOPA melanin would indicate that each isochromat, regardless of electronic transition energy, contains the same distribution of IR-active functional groups. However, it is difficult to imagine how chromophores that differ in size, as suggested by wavelength-dependent Raman scattering results ${ }^{11}$, could all have a common IR absorption spectrum. Furthermore, if the chromophores were to remain constant in size, then changes in functional groups would be needed to tune the electronic transition energy, but this would lead to unique IR signatures for different isochromats contrary to the observations.

We propose that coupling among nearby protomolecules in eumelanin provides a natural way to understand how the members of an isochromat collectively give rise to a common vibrational spectrum. $\mathrm{TEM}^{13}$ and transient absorption ${ }^{14}$ measurements support the model that eumelanin is made of $\pi$ stacked nanoaggregates ${ }^{32}$. Closely spaced chromophores can couple electronically causing new transition energies that differ from those of the separated chromophores. We propose that photoexcitation of multiple coupled eumelanin chromophores produces Frenkel and/or charge transfer (CT) excitons that bleach vibrations associated with each chromophore, similar to how electronically exciting $\pi$-stacked nucleobase dimers bleaches the vibrational fundamentals of both bases ${ }^{24}$. Bleaching of the vibrational bands of multiple interacting molecules is also illustrated by the TRIR spectrum of the concentrated Cat+Quin mixture, where preferential excitation of Quin in Cat:Quin complexes leads to bleaching of vibrational modes of both Cat and Quin molecules (Fig. 2f) ${ }^{21}$.

Chromophore size, electronic coupling, and the presence of redox-active functional groups like quinones are all likely to tune the transition energy of a chromophore aggregate. Disentangling their relative contributions is difficult, but because every isochromat contains all possible functional groups, functional group content (quinone, semiquinone, hydroquinone, etc.) is likely less important than size for determining electronic transition energies. Tuna et al. showed in a computational study that increasing the size of a DHI oligomer from 1 to 5 monomer units shifts its lowest transition energy by $\sim 1 \mathrm{eV}^{16}$. Such a shift could tune the energies of eumelanin chromophores through much of the visible spectrum (the energy difference between 400 and 600 $\mathrm{nm}$ is $\sim 1 \mathrm{eV}$ ).

This conclusion is further supported by wavelengthdependent resonance Raman spectra of eumelanins ${ }^{33}$, including DOPA melanin ${ }^{11}$, which point to the presence of multiple types of chromophores that differ in electronic conjugation length, or $\mathrm{sp}^{2}$ domain size ${ }^{11}$. It is important to note that by size we are referring to the conjugation length, which could differ from the physical size of a protomolecule. Instead of tuning transition energies, functional groups could carve out $\mathrm{sp}^{2}$ domains of different size as they do in graphene oxide ${ }^{34}$. Alternatively, in aggregates of diverse chromophores, the random presence of several possible functional groups that are equally likely to be reduced or oxidized could cause shifts in transition energies that average to near zero, allowing chromophore size to be the primary factor behind the variation in electronic transition energy.

The time-evolution of the TRIR spectra provide additional insights into the ultrafast relaxation of excited eumelanin chromophores. The TRIR spectral dynamics and kinetics in Fig. 5 reveal that the majority of the population of electronic ground states recovers on the early picosecond timescale, consistent with electronic bleach recovery kinetics ${ }^{11,35}$. TRIR spectroscopy probes thermal dissipation directly by monitoring the growth in the thermal signal of $\mathrm{D}_{2} \mathrm{O}$, which is complete by $\sim 20$ ps. Note that the roughly simultaneous rise of the thermal signal and the decay of the DOPA melanin signal (Fig. 5d) indicate that heat is transferred to the solvent immediately following relaxation of the excited state population. Previous measurements of ultralow photoluminescence quantum yields in eumelanin established the dominance of nonradiative deactivation pathways ${ }^{36}$, which were shown to be ultrafast in pioneering measurements by Simon and co-workers ${ }^{37}$. Although photoacoustic calorimetry experiments were used to infer that these pathways transfer heat to the solvent on a sub-nanosecond timescale ${ }^{38}$, our measurements unambiguously show that heat is rapidly transferred from the DOPA melanin to the solvent via vibrational energy transfer in several picoseconds.

The TRIR spectra mirror much of the structure seen in the inverted FTIR spectrum as expected for bleaching signals, but the $\Delta A$ signals are positive everywhere except between 1600 and $1650 \mathrm{~cm}^{-1}$ (Fig. 4). To maintain the near mirror-image symmetry with the inverted FTIR spectrum, a positive signal contribution that varies slowly with probe frequency must be present. The positive TRIR signals seen above $1750 \mathrm{~cm}^{-1}$ occur at frequencies where there is little to no absorption by DOPA melanin in its ground electronic state (see the FTIR spectrum in Fig. 4). We propose that the signal here, which is broader than for typical excited state vibrations, is electronic in origin. The average signal decay measured between $1750-1900 \mathrm{~cm}^{-1}$ exhibits stretched exponential kinetics that at every excitation wavelength match the decay kinetics seen at near-infrared probe wavelengths (see Supplementary Fig. 3) $^{11}$. The kinetics at $1310 \mathrm{~cm}^{-1}$ (Fig. 5e), which are free of the $\mathrm{D}_{2} \mathrm{O}$ thermal signal (Supplementary Fig. 4), track with the fast decay component of the near-infrared kinetics, except at times $<0.5 \mathrm{ps}$ (Supplementary Fig. 3). The matching kinetic behavior indicates that the electronic state responsible for the mid-IR signals is the same one detected in the near-infrared ${ }^{11}$. 
The identical TRIR kinetic traces in Fig. 5e indicate that regardless of which set of DOPA melanin chromophores is photoexcited, excited state relaxation proceeds at the same rate. Because every isochromat contains all possible IR-active functional groups, we propose that CT between reduced and oxidized indolic units within chromophores ${ }^{20}$ or between chromophores in aggregates creates CT excitons or polaron pairs (PPs) that decay with common deactivation kinetics ${ }^{11}$. The observation here of an electronic absorption signal in the mid-IR that matches the near-IR transient absorption kinetics we reported recently ${ }^{11}$ supports this assignment. Many computational studies to date consider stacks of chromophores with a fixed redox state ${ }^{15,16}$, and future calculations of coupled chromophores with mixed redox states are needed.

Photoinduced electronic absorption in the mid-IR by polarons and PPs formed via CT is a hallmark of semiconducting polymers $^{39}$. Ultrafast (sub-picosecond) generation of geminate PPs in polymers ${ }^{40,41}$, such as poly(3-hexylthiophene) $(\mathrm{P} 3 \mathrm{HT})(<20 \mathrm{fs})^{42}$, is reminiscent of our findings in DOPA melanin. Geminate PPs show excitation power-independent decay kinetics ${ }^{39,41,43}$ because they decay unimolecularly ${ }^{41,44}$. These observations align well with the excitation power-independent TRIR kinetics at $1310 \mathrm{~cm}^{-1}$ of DOPA melanin (Fig. 5f). Furthermore, contacts between reduced and oxidized structures in DOPA melanin can facilitate the CT needed for polaron formation. For example, photoexciting either molecule in the Cat:Quin heterodimer induces an ultrafast comproportionation reaction yielding a semiquinone radical pair state $^{21}$.

Ultrafast recombination of PPs in eumelanin has not been proposed previously and can explain the electronic deactivation responsible for photoprotection. PPs that fail to recombine could lead to the long-lived radical species, such as those detected by electron spin resonance in photoexcited natural and synthetic eumelanins ${ }^{45}$. This proposal differs from the previous assignment of ultrafast nonradiative decay in non-aggregated DHICA oligomers to singlet excited state deactivation ${ }^{46}$. Aggregation of eumelanin subunits enables the ultrafast CT needed to form PPs. Altogether, the TRIR results suggest that the photogenerated species in eumelanin differ strongly from those in non-aggregated model systems, suggesting the importance of considering alternative ultrafast deactivation mechanisms to explain eumelanin photoprotection.

In conclusion, the present UVF results unexpectedly reveal that excited groups of chromophores in a synthetic eumelanin selected by their transition energies have a common TRIR spectrum and decay kinetics. The insight that all chromophore subsets contain the same distribution of functional groups is explained by a model in which chromophores decorated at random with all possible IR-active functional groups are electronically coupled together in aggregates, leading to wavelength-independent excited state relaxation. TRIR spectroscopy directly detects ultrafast thermal dissipation from the relaxed eumelanin chromophores to the solvent and reveals that the photoexcited states in eumelanin may have different electronic character than in monomers and oligomers. Finally, the UVF method can be applied effectively to the study of other heterogeneous carbonaceous systems such as graphene oxide ${ }^{47}$, carbon nanodots ${ }^{48}$, chromophoric dissolved organic matter ${ }^{49}$, and pitch $^{50}$, all of which feature elusive combinations of chromophores and eumelanin-like absorption.

\section{Methods}

Materials. DOPA melanin was synthesized from L-DOPA using base-initiated oxidative polymerization ${ }^{11}$. First, $1 \mathrm{~g}$ of L-DOPA (Sigma Aldrich, $\geq 98 \%$ ) was mixed with $200 \mathrm{~mL}$ of nanopure water. Then ammonium hydroxide solution
(28-30\% by mass, Fisher Chemical) was added to the mixture dropwise until the $\mathrm{pH}$ was stabilized at 9.5. At this time, the L-DOPA was fully dissolved. The mixture was gently stirred, and air was bubbled for 3 days. Afterward, approximately $200 \mathrm{~mL}$ of acetonitrile was added to the reaction mixture, causing the DOPA melanin product to flocculate. The reaction mixture was then centrifuged at $10,000 \times g$, yielding a pellet of the DOPA melanin. After removing the supernatant, the pellet was resuspended in fresh acetonitrile. Centrifugation and resuspension were repeated 2-3 times until the supernatant was clear and colorless and the UV-vis spectrum of the supernatant lacked absorption bands above $250 \mathrm{~nm}$. Finally, the pellet was dried under nitrogen, yielding dark and shiny flakes with high water solubility.

For FTIR and TRIR measurements, DOPA melanin powder was dissolved at $5 \mathrm{mg} \mathrm{mL}^{-1}$ in phosphate-buffered $\mathrm{D}_{2} \mathrm{O}$ solution, containing $25 \mathrm{~mm}$ each of sodium phosphate monobasic and sodium phosphate dibasic. The $\mathrm{pD}$ value of the buffer and DOPA melanin solution was $7.6 \pm 0.1$, which is close to what is expected in the stratum spinosum and stratum basale in human skin, where melanocytes produce melanins ${ }^{51}$

The Cat and Quin derivatives, 3,5-di-t-butylcatechol and 3,5-di-t-butyl-oquinone, respectively, were purchased from Sigma Aldrich ( $98 \%$ purity). Cyclohexane was from Acros Organics (HPLC grade, 99.8\%). The dilute Cat and Quin mixture contained Cat and Quin concentrations of $5 \mathrm{~mm}$ and $1 \mathrm{~mm}$, respectively. These concentrations were increased tenfold in the concentrated Cat and Quin mixture. Control samples were studied for each mixture, in which each component (Cat or Quin) was present by itself in cyclohexane solution at the same concentration as in each mixture.

Steady-state spectroscopy. UV-vis absorption spectra were measured using a Cary UV/Vis/NIR spectrometer (Agilent; Santa Clara, CA) set to a $2 \mathrm{~nm}$ effective spectral bandwidth. Solutions were measured either in quartz cuvettes or a homemade demountable liquid cell consisting of Teflon spacers and $\mathrm{CaF}_{2}$ windows. Pathlengths were selected to ensure that the maximum absorbance did not exceed 1.

IR absorption spectra were recorded using an FTIR spectrometer (FT/IR-4200, JASCO; Easton, MD). The spectral resolution was set to $4 \mathrm{~cm}^{-1}$ for DOPA melanin and $0.5 \mathrm{~cm}^{-1}$ for the Cat and Quin solutions. Solutions were measured in the same demountable liquid cell described above using a $100 \mu \mathrm{m}$ spacer for $\mathrm{D}_{2} \mathrm{O}$ solutions and $200 \mu \mathrm{m}$ or $500 \mu \mathrm{m}$ spacers for cyclohexane solutions. For DOPA melanin measurements, special care was taken to avoid spurious signals arising from unequal concentrations of HOD and buffer between blank and sample scans.

TRIR spectroscopy. TRIR spectroscopy was performed using a commercial transient 2D IR spectrometer (2DQuick IR with the 2DQuick Transient add-on module PhaseTech Spectroscopy; Madison, WI). The ultrafast UV-vis and mid-IR pulses were generated using optical parametric amplifiers (OPAs). Specifically, the output of a Ti:Sapphire laser amplifier (Astrella, Coherent; Santa Clara, CA) was split and sent into an OPA (TOPAS Prime, Coherent; Santa Clara, CA) to generate UV or visible excitation pulses and another OPA with a difference frequency generation (DFG) attachment (TOPAS Prime/DFG, Coherent; Santa Clara, CA) to generate mid-infrared probe pulses. For each excitation wavelength used $(265,300,400,500$, and $600 \mathrm{~nm}$ ), the on-axis excitation fluence was between 0.5 and $1 \mathrm{~mJ} \mathrm{~cm}^{-2}$, assuming a Gaussian beam. For power-dependent measurements performed with $500 \mathrm{~nm}$ excitation, the on-axis fluence was in the range of $0.4-5 \mathrm{~mJ} \mathrm{~cm}^{-2}$. The magic angle pump-probe condition was used in all cases. The sample pathlength was $100 \mu \mathrm{m}$ and the samples were continuously recirculated during all scans. We monitored the absorption spectrum of the solution after each scan to ensure there was no sample degradation.

Because the spectral full-width at half-maximum of the mid-IR pulses was limited to at most $\sim 175 \mathrm{~cm}^{-1}$, measurements were made piecewise from $\sim 1300-1900 \mathrm{~cm}^{-1}$ for DOPA melanin solutions. The three regions measured were (1) $1290-1475 \mathrm{~cm}^{-1}$, (2) $1450-1700 \mathrm{~cm}^{-1}$, and (3) $1675-2000 \mathrm{~cm}^{-1}$. We took special care in properly calibrating the detected mid-IR frequency axis for each of these regions using the IR absorption lines of reference materials. For region 1, we used tetrahydrofuran, unstretched Parafilm, and a plastic film. For region 2, we used water vapor. For region 3, water vapor and trimethylamine were used.

For TRIR measurements on the Cat and Quin solutions, the on-axis excitation fluence was $\sim 2 \mathrm{~mJ} \mathrm{~cm}^{-2}$ at all excitation wavelengths, assuming a Gaussian beam. Note that a broad $\Delta A$ signal, spanning the entire spectral window, is observed when exciting the dilute Cat+Quin mixture at $280 \mathrm{~nm}$ (Supplementary Fig. 8). This electronic background signal, which is observed when using UV pulses at high excitation fluence, was removed from the data as reported previously ${ }^{52}$, and as described in the Supplementary Note 6.

\section{Data availability}

All experimental data presented in the main text and in Supplementary Information are available from the corresponding author upon request.

Received: 22 July 2020; Accepted: 18 August 2020;

Published online: 11 September 2020 


\section{References}

1. Ju, K.-Y. et al. Unraveling the molecular nature of melanin changes in metastatic cancer. J. Biomed. Opt. 24, 051414 (2019).

2. Sarna, M., Krzykawska-Serda, M., Jakubowska, M., Zadlo, A. \& Urbanska, K. Melanin presence inhibits melanoma cell spread in mice in a unique mechanical fashion. Sci. Rep. 9, 9280 (2019).

3. Zecca, L. et al. Substantia nigra neuromelanin: structure, synthesis, and molecular behaviour. Mol. Pathol. 54, 414-418 (2001).

4. Ajjan, F. N., Mecerreyes, D. \& Inganäs, O. Enhancing energy storage devices with biomacromolecules in hybrid electrodes. Biotechnol. J. 14, 1900062 (2019).

5. Xu, R., Gouda, A., Caso, M. F., Soavi, F. \& Santato, C. Melanin: a greener route to enhance energy storage under solar light. ACS Omega 4, 12244-12251 (2019).

6. Huang, L. et al. Recent advances and progress on melanin-like materials and their biomedical applications. Biomacromolecules 19, 1858-1868 (2018).

7. Eom, T. et al. Naturally derived melanin nanoparticle composites with high electrical conductivity and biodegradability. Part Part Syst. Charact. 36, 9 (2019).

8. Migliaccio, L. et al. Evidence of unprecedented high electronic conductivity in mammalian pigment based eumelanin thin films after thermal annealing in vacuum. Front Chem. 7, 162 (2019).

9. d'Ischia, M., Napolitano, A., Pezzella, A., Meredith, P. \& Buehler, M. Melanin biopolymers: tailoring chemical complexity for materials design. Angew. Chem. Int Ed. 59, 11196-11205 (2020).

10. Tran, M. L., Powell, B. J. \& Meredith, P. Chemical and structural disorder in eumelanins: a possible explanation for broadband absorbance. Biophys. J. 90, 743-752 (2006).

11. Kohl, F. R., Grieco, C. \& Kohler, B. Ultrafast spectral hole burning reveals the distinct chromophores in eumelanin and their common photoresponse. Chem. Sci. 11, 1248-1259 (2020).

12. Thompson, A. et al. Dual-wavelength pump-probe microscopy analysis of melanin composition. Sci. Rep. 6, 36871 (2016).

13. Watt, A. A. R., Bothma, J. P. \& Meredith, P. The supramolecular structure of melanin. Soft Matter 5, 3754-3760 (2009).

14. Ju, K.-Y., Fischer, M. C. \& Warren, W. S. Understanding the role of aggregation in the broad absorption bands of eumelanin. ACS Nano 12, 12050-12061 (2018).

15. Chen, C. T. et al. Excitonic effects from geometric order and disorder explain broadband optical absorption in eumelanin. Nat. Commun. 5, 3859 (2014).

16. Tuna, D., Udvarhelyi, A., Sobolewski, A. L., Domcke, W. \& Domratcheva, T. Onset of the electronic absorption spectra of isolated and pi-stacked oligomers of 5,6-dihydroxyindole: an ab initio study of the building blocks of eumelanin. J. Phys. Chem. B 120, 3493-3502 (2016).

17. Stark, K. B. et al. Effect of stacking and redox state on optical absorption spectra of melanins-comparison of theoretical and experimental results. $J$. Phys. Chem. B 109, 1970-1977 (2005).

18. Pezzella, A. et al. Disentangling eumelanin "black chromophore": visible absorption changes as signatures of oxidation state- and aggregationdependent dynamic interactions in a model water-soluble 5,6-dihydroxyindole polymer. J. Am. Chem. Soc. 131, 15270-15275 (2009)

19. Prampolini, G., Cacelli, I. \& Ferretti, A. Intermolecular interactions in eumelanins: a computational bottom-up approach. I. small building blocks. RSC Adv. 5, 38513-38526 (2015).

20. Matta, M., Pezzella, A. \& Troisi, A. Relation between local structure, electric dipole, and charge carrier dynamics in DHICA melanin: a model for biocompatible semiconductors. J. Phys. Chem. Lett. 11, 1045-1051 (2020).

21. Grieco, C., Empey Jennifer, M., Kohl, F. R. \& Kohler, B. Probing eumelanin photoprotection using a catechol:quinone heterodimer model system. Faraday Discuss 216, 520-537 (2019).

22. Centeno, S. A. \& Shamir, J. Surface enhanced Raman scattering (SERS) and FTIR characterization of the sepia melanin pigment used in works of art. J. Mol. Struct. 873, 149-159 (2008).

23. Powell, B. J. et al. A first-principles density-functional calculation of the electronic and vibrational structure of the key melanin monomers. J. Chem. Phys. 120, 8608-8615 (2004).

24. Zhang, Y. et al. Efficient UV-induced charge separation and recombination in an 8-oxoguanine-containing dinucleotide. Proc. Natl Acad. Sci. USA 111 11612-11617 (2014).

25. Jankowiak, R. \& Small, G. J. Hole-burning spectroscopy and relaxation dynamics of amorphous solids at low temperatures. Science 237, 618-625 (1987).

26. Song, Y. et al. Multispectral multidimensional spectrometer spanning the ultraviolet to the mid-infrared. Rev. Sci. Instrum. 90, 013108 (2019).

27. Oliver, T. A. A. Recent advances in multidimensional ultrafast spectroscopy. R. Soc. Open Sci. 5, 171425 (2018).

28. Oliver, T. A. A., Lewis, N. H. C. \& Fleming, G. R. Correlating the motion of electrons and nuclei with two-dimensional electronic-vibrational spectroscopy. Proc. Natl Acad. Sci. USA 111, 10061-10066 (2014).
29. Hamm, P., Lim, M. \& Hochstrasser, R. M. Structure of the amide I band of peptides measured by femtosecond nonlinear-infrared spectroscopy. J. Phys Chem. B 102, 6123-6138 (1998).

30. Shim, S.-H., Strasfeld, D. B., Ling, Y. L. \& Zanni, M. T. Automated 2D IR spectroscopy using a mid-IR pulse shaper and application of this technology to the human islet amyloid polypeptide. Proc. Natl Acad. Sci. USA 104, 14197-14202 (2007).

31. Cervetto, V., Helbing, J., Bredenbeck, J. \& Hamm, P. Double-resonance versus pulsed Fourier transform two-dimensional infrared spectroscopy: An experimental and theoretical comparison. J. Chem. Phys. 121, 5935-5942 (2004).

32. Chen, C.-T. et al. Self-assembly of tetramers of 5,6-dihydroxyindole explains the primary physical properties of eumelanin: experiment, simulation, and design. ACS Nano 7, 1524-1532 (2013).

33. Huang, Z. et al. Raman spectroscopy of in vivo cutaneous melanin. J. Biomed Opt. 9, 1198-1205 (2004)

34. Loh, K. P., Bao, Q. L., Eda, G. \& Chhowalla, M. Graphene oxide as a chemically tunable platform for optical applications. Nat. Chem. 2, 1015-1024 (2010).

35. Simpson, M. J. et al. Near-infrared excited state dynamics of melanins: the effects of iron content, photo-damage, chemical oxidation, and aggregate size. J. Phys. Chem. A 118, 993-1003 (2014).

36. Nighswander-Rempel, S. P., Riesz, J., Gilmore, J. \& Meredith, P. A quantum yield map for synthetic eumelanin. J. Chem. Phys. 123, 6 (2005).

37. Nofsinger, J. B., Ye, T. \& Simon, J. D. Ultrafast nonradiative relaxation dynamics of eumelanin. J. Phys. Chem. B 105, 2864-2866 (2001).

38. Forest, S. E. \& Simon, J. D. Wavelength-dependent photoacoustic calorimetry study of melanin. Photochem. Photobiol. 68, 296-298 (1998)

39. Jiang, X. M. et al. Spectroscopic studies of photoexcitations in regioregular and regiorandom polythiophene films. Adv. Funct. Mater. 12, 587-597 (2002)

40. Stallhofer, K. et al. Dynamics of short-lived polaron pairs and polarons in polythiophene derivatives observed via infrared-activated vibrations. J. Phys Chem. C. 123, 28100-28105 (2019).

41. Guo, J., Ohkita, H., Benten, H. \& Ito, S. Near-IR femtosecond transient absorption spectroscopy of ultrafast polaron and triplet exciton formation in polythiophene films with different regioregularities. J. Am. Chem. Soc. 131, 16869-16880 (2009)

42. De Sio, A. et al. Tracking the coherent generation of polaron pairs in conjugated polymers. Nat. Commun. 7, 13742 (2016).

43. Klimov, V. I., McBranch, D. W., Barashkov, N. N. \& Ferraris, J. P. Femtosecond dynamics of excitons in $\pi$-conjugated oligomers: the role of intrachain two-exciton states in the formation of interchain species. Chem Phys. Lett. 277, 109-117 (1997).

44. Ohkita, H. \& Ito, S. Transient absorption spectroscopy of polymer-based thin film solar cells. Polymer 52, 4397-4417 (2011)

45. Sarna, T. \& Sealy, R. C. Free radicals from eumelanins: quantum yields and wavelength dependence. Arch. Biochem. Biophys. 232, 574-578 (1984).

46. Corani, A. et al. Superior photoprotective motifs and mechanisms in eumelanins uncovered. J. Am. Chem. Soc. 136, 11626-11635 (2014).

47. Georgakilas, V. et al. Noncovalent functionalization of graphene and graphene oxide for energy materials, biosensing, catalytic, and biomedical applications. Chem. Rev. 116, 5464-5519 (2016).

48. Xiao, L. \& Sun, H. Novel properties and applications of carbon nanodots. Nanoscale Horiz. 3, 565-597 (2018).

49. Nelson, N. B. \& Siegel, D. A. The global distribution and dynamics of chromophoric dissolved organic matter. Annu. Rev. Mar. Sci. 5, 447-476 (2013)

50. Chen, P. et al. Petroleum pitch: exploring a 50-year structure puzzle with realspace molecular imaging. Carbon 161, 456-465 (2020).

51. Koch, A. \& Schwab, A. Cutaneous $\mathrm{pH}$ landscape as a facilitator of melanoma initiation and progression. Acta Physiol. 225, e13105 (2019).

52. Grieco, C. et al. Intermolecular hydrogen bonding modulates $\mathrm{O}-\mathrm{H}$ photodissociation in molecular aggregates of a catechol derivative. Photochem Photobiol. 95, 163-175 (2019)

\section{Acknowledgements}

This work was supported by a grant by NSF (CHE-1800471) and by startup funding from The Ohio State University.

\section{Author contributions}

C.G. and F.R.K. designed experiments. C.G. performed all measurements, analyzed the data, and created the figures. F.R.K. synthesized and purified the DOPA melanin samples. A.T.H. contributed to concepts underlying UVF. C.G. and B.K. wrote the manuscript. B.K. directed the research.

\section{Competing interests}

There is no competing interest to declare. 


\section{Additional information}

Supplementary information is available for this paper at https://doi.org/10.1038/s41467020-18393-w.

Correspondence and requests for materials should be addressed to B.K.

Peer review information Nature Communications thanks the anonymous reviewers for their contribution to the peer review of this work.

Reprints and permission information is available at http://www.nature.com/reprints

Publisher's note Springer Nature remains neutral with regard to jurisdictional claims in published maps and institutional affiliations. (c) (i) Open Access This article is licensed under a Creative Commons Attribution 4.0 International License, which permits use, sharing, adaptation, distribution and reproduction in any medium or format, as long as you give appropriate credit to the original author(s) and the source, provide a link to the Creative Commons license, and indicate if changes were made. The images or other third party material in this article are included in the article's Creative Commons license, unless indicated otherwise in a credit line to the material. If material is not included in the article's Creative Commons license and your intended use is not permitted by statutory regulation or exceeds the permitted use, you will need to obtain permission directly from the copyright holder. To view a copy of this license, visit http://creativecommons.org/ licenses/by/4.0/.

(C) The Author(s) 2020 